\title{
Traffic Control System and Technologies: A Survey
}

\author{
Anand Shah \\ Department of Computer Engineering \\ Sinhgad College of Engineering \\ (affiliated to Savitribai Phule Pune University) \\ Pune, India \\ Rohit Patil \\ Department of Computer Engineering \\ Sinhgad College of Engineering \\ (affiliated to Savitribai Phule Pune University) \\ Pune, India
}

\author{
Vishal Sisode \\ Department of Computer Engineering \\ Sinhgad College of Engineering \\ (affiliated to Savitribai Phule Pune University) \\ Pune, India \\ Sahil Gogave \\ Department of Computer Engineering \\ Sinhgad College of Engineering \\ (affiliated to Savitribai Phule Pune University) \\ Pune, India
}

\author{
Prof. J. B. Kulkarni \\ Assistent Professor Department of Computer Engineering \\ Sinhgad College of Engineering (affiliated to Savitribai Phule Pune University) \\ Pune, India
}

\begin{abstract}
The rapid increase in vehicles and large time delays between traffic lights have been the major reason behind the cost of congestion. To overcome this problem, several techniques and methods have been introduced throughout the years which are trying to make traffic control systems dynamic. The conventional traffic control systems work on fixed timings allocated to each side of the junction which cannot be altered, In smart traffic control systems, these timings are controlled according to traffic density. The purpose of this study is to investigate whether these methods are feasible and if we can find any correlations between them. Methods discussed in this paper include the use of IR sensors, RFID tags, Thermal cameras and Reinforcement learning.
\end{abstract}

Index Terms-RFID, IR sensors, Embedded Systems, Reinforcement Learning, SURTRAC

\section{INTRODUCTION}

When the traffic systems were firstly introduced to the world, they incorporated a system of open loop control. This system was quite basic. It had the advantages such as:

1) Centralized control

2) Large number of implementable plans

3) Easy detection of malfunction in the traffic control system

Since there are trends in movement of centralized to decentralized infrastructure, development of smart and intelligent devices, increase in population leading to increased vehicular population, this system can't take care of so many changes at once. Without any sort of feedback, there is no report on the effective working of the system nor the disadvantages of the signal plans that have been implemented. In today's age, there is a need for dynamic control in everything considering traffic signals as well. This is the basic characteristic lacked by open loop control system which only implements signal timings according to devised plans rather than conforming to the current conditions.

In order to account feedback into the system, closed loop control systems where brought in. According to the traffic conditions, the optimal plan are implemented into the system by selecting one from the libraries of plans considering the pattern similar to the observed pattern or judging by the traffic conditions, the plans are made on-line. These plans take about 3 - 5 minutes to generate and be used.

The drawback in closed loop system arises in the development and implementation of plans which takes about 3-5 minutes wherein whole traffic scenario can change in that amount of time. Apart from that, the plans also were expensive to prepare and maintain. So there arose a need for adaptive systems which could implement changes in matter of seconds.

\section{LITERATURE SURVEY}

In order to construct an adaptive system, the system needs to take feedback from the environment into account. Various techniques and methods can be used to do so as well as systems have to be look through for this purpose. Area traffic control systems like SCOOT(Split, Cycle, Offset Optimisation Technique), SCATS(Sydney Coordinated Adaptive Traffic System) and OPAC(Optimized Policies for Adaptive Control) which have been proposed in the 20th century can reduce traffic delays, fuel consumption, accident, congestion, travel time, environmental pollution substantially and can increase average flow speed. SCOOT, SCAT and OPAC are popular in advanced countries but such systems cannot cope up with Indian situations because in India traffic is not lane following, highly mixed traffic, uncontrolled side road and on-street 
parking, data loss due to power failure and availability of funds.

Jasmine Rani L. et al. [1] have proposed the use of IR(Infrared) sensors in order to collect vehicle data. The flaws appear when multiple vehicles arrive in the scene. This would require many IR sensors to compute the traffic density and with that, the data obtained may not be as reliable. Also the categorization of vehicle on the basis of IR sensor data is very difficult to accomplish. This makes the use of IR sensors unfeasible. As specified in the papers, the traffic lights are dependent on the signals received from IR sensors, if the IR sensors are occupied by a single vehicle or multiple vehicles in a less congested traffic lane, their system might give more importance to a less congested area causing more congestion. Moving forward to use of RFID (Radio Frequency Identi- fication) for the traffic control problem, Al-Khateeb et. al. [2] have developed a system which senses the situation through RFID devices. Major requirement of that system is to have RFID tags which will transmit data. The process of having every vehicle tagged is long lasting and difficult to accomplish since vehicles which were manufactured before 2017(in India) didn't mandatorily consist of RFID tags.

Thermal cameras could aid in sensing vehicle inflow during conditions with low visibility. This advantage comes with a hefty cost. P. Jagannadha Rao et. al. [3] have suggested the idea to use them as detectors. Despite the advantages, when we consider the working of thermal cameras during times when visibility is okay, normal cameras will outperform them while also being relatively cheaper than them. From the plethora of data sources available, it will be easier to collect and harness images about non-thermal cameras than thermal cameras in order to build a model from them.

While the devices mentioned above do offer partial solutions for traffic control, it is also important as to where the sensors and devices would be placed and mounted. Undeniably, embedded systems provide cost efficient solutions to computation at nodes while also being structurally small and providing most of the functionalities that a modern computer can provide whilst also accommodating the said equipment. Sk Riyaz Hussain, et al [4] in their paper have considered the idea of monitoring traffic density through Raspberry Pi. They have measured the density of traffic by differentiation between white and black pixels. This method would not prove useful when a large vehicle like a truck or a bus or a number of them takes up the most of the camera footage.

Although the weakness, one takeaway is that an embedded system can prove as a useful device going forward because of the functionalities offered at limited cost.

Maram Bani Younes and Azzedine Boukerche [5] have offered insights into using VANETs(Vehicular Ad-hoc Networks) technology to develop an intelligent traffic light controlling algorithms by placing wireless transceiver and GPS tools on vehicles. Each vehicle over the road periodically broadcasts the basic traveling data (i.e., location, speed, direction, destination, etc). Vehicles receive the basic traffic data of surrounding vehicles. This will just increase cost of the system because vehicles will require to be equipped with the above mentioned devices as well as the computations that will be performed may take a lot of time in congested areas because of mass amount of availability of vehicles. Also the reports

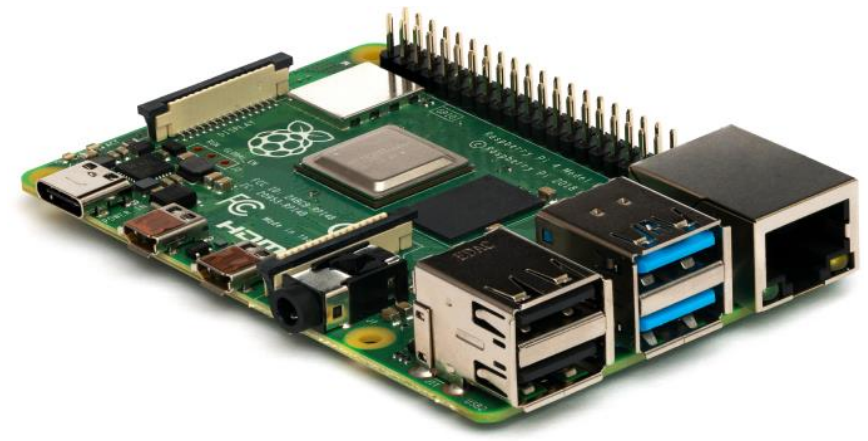

Fig. 1. Raspberry Pi board

that the agent will receive may be incoherent depending on different reporting vehicle. Also the system is dependent on vehicles to pass the information and may not work when and if the devices stop functioning.

Looking at speed of the vehicles in order to judge traffic is an interesting perspective put forward by Li Jinyang et. al. [6]. This can be implemented by V2I(Vehicle to Infrastructure) where road vehicles interact with the road infrastructure/system. The main issue still stands in the vehicles tailored with the devices to do so. Also while vehicle speed though may prove out to show the real time traffic conditions, if certain vehicle drivers are used to driving slowly, the performance of the proposed model may be hindered.

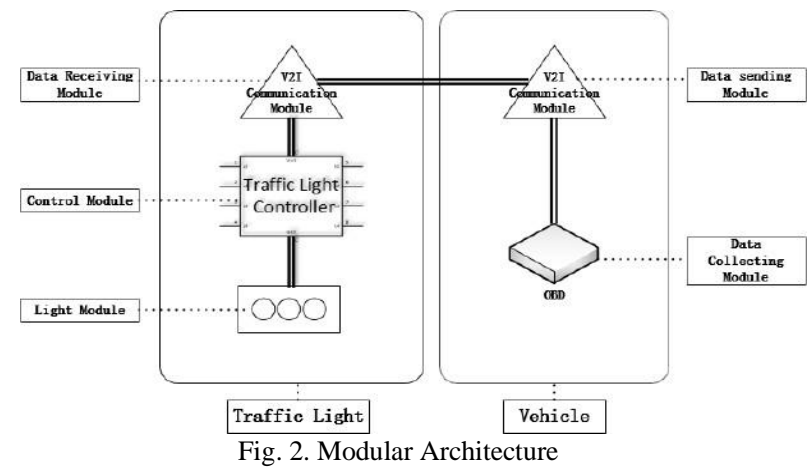

With the growing popularity of RL(Reinforcment Learning) in recent history, it brings about a new dynamic whenever the question of an adaptive/ learning system comes in the discussion. RL uses Q-Learning to do so which constructs a key-value pair between states and actions which will lead to the optimal calculated reward. Similar approach can be used for traffic as has been experimented by El-Tantawy et. al. [7]. The problem within this lies in the fact that it is not possible to store so many states because of memory constraints and the vast amount of variables that need to be accounted.

Wei Hua et al. [8] in their proposed system known as 


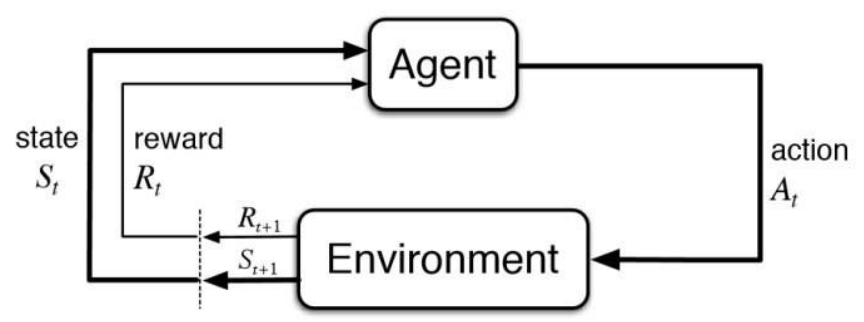

Fig. 3. Reinforcement Learning

Intellilight have found out that Deep RL overcomes the constraints in RL while offering better results in hindsight.

SURTRAC(Scalable Urban Traffic Control) is one of the recent developments into solving the problem of traffic bottleneck developed by Stephen F. Smith, et al. [9]. It uses various video detectors to detect vehicle and their movements which are used to manage the traffic lights accordingly. Their system has improved traffic flow efficiency drastically at the tests conducted in their pilot site. But in a country like India where lane discipline is not mandatory, this system may not be as effective.

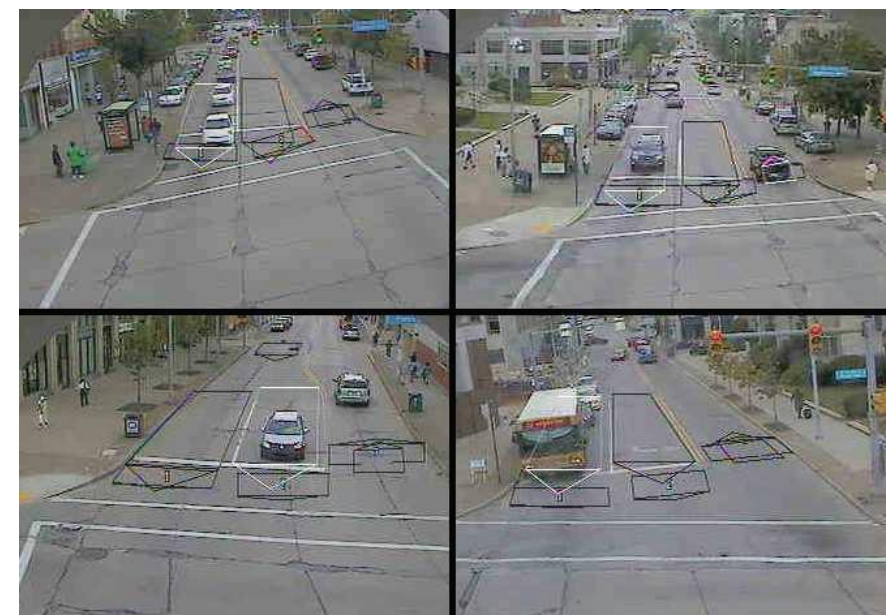

Fig. 4. The placement of detectors in a typical installation(SURTRAC)

\section{PROPOSED METHODOLOGY}

Hereby, the following system is being proposed wherein the traffic conditions will be monitored through cameras. The cameras will be input to the next module which will carry out the object detection. This output will then be forwarded to the learning algorithm and on the basis of the analyzing carried out by the algorithm, the system will propose new time slots/schedules that need to be implemented by the traffic system. This system will not alter the sequential switching flow from one signal to another that is already in existence.

The system will monitor the traffic flows that pass through the road intersection and will work in a decentralized manner.

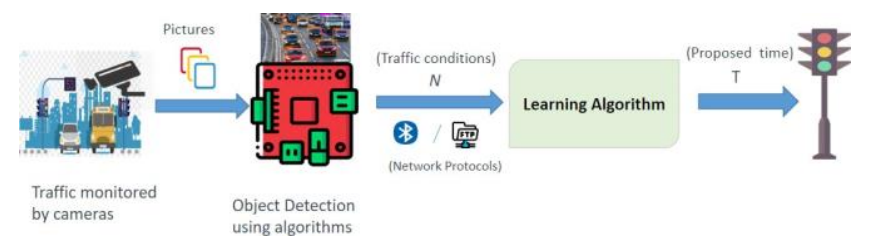

Fig. 5. New proposed system

\section{CONCLUSION}

From the existing technologies that were surveyed, cameras seem to be the most efficient and best method in order to capture the traffic conditions. IR and thermal sensors are not as effective as cameras and may lead to inconsistent results. The tests which were performed in [3] with thermal cameras operated in static lab environment and minimal dynamic objects in simulated environment which cannot showcase the actual traffic conditions. Embedded systems provide vital on node computing at cheap costs and can also be used as a medium to communicate with traffic signals.

VANET and RFID based system require the vehicles to send information. Without a vehicle capable of doing so, or any minor inconvenience dwindling their ability to do so, the results might not be as accurate. Reinforcement learning is a widely suggested solution for traffic control, but the constraints that come along with it does not make it the best candidate to solve the problem. However, deep reinforcement learning could help overcome those issues.

SURTRAC converts the traffic problem into a single machine scheduling problem. It definitely has shown promising results at the test site. Since it services on the vehicle to vehicle basis, it may alter cyclic scheduling of traffic lights. Thereby, this may be problematic in a country like India.The following results are taken from [9] :

\begin{tabular}{|l|l|l|l|l|l|l|}
\hline Percent Improvement & Average Vehicles & Travel Time & Speed & Number of Stops & Wait Time & Emissions \\
\hline AM rush & 5228 & $30.11 \%$ & $33.78 \%$ & $29.14 \%$ & $47.78 \%$ & $23.83 \%$ \\
\hline Mid day & 8007 & $32.83 \%$ & $48.55 \%$ & $52.58 \%$ & $49.82 \%$ & $29.00 \%$ \\
\hline PM rush & 9548 & $22.65 \%$ & $27.45 \%$ & $8.89 \%$ & $35.60 \%$ & $18.41 \%$ \\
\hline Evening & 7157 & $17.52 \%$ & $27.81 \%$ & $34.97 \%$ & $27.56 \%$ & $14.01 \%$ \\
\hline Overall & 29940 & $25.79 \%$ & $34.02 \%$ & $31.34 \%$ & $40.64 \%$ & $21.48 \%$ \\
\hline
\end{tabular}

\section{ACKNOWLEDGMENT}

The undertaking and progress of this project required a lot of guidance and assistance from many people and we are extremely privileged to have got this all along the completion of this project report. All that we have done is only due to such supervision and assistance and we would not forget to thank them.

We respect and thank Prof. J.B.Kulkarni, for providing us an opportunity and giving us all support and guidance which made us complete the project report duly. We are extremely thankful to her for providing such nice support and guidance.

We owe our deep gratitude to Prof.M.P.Wankhade, HOD Department of Computer Engineering, who took keen interest in our project work, for providing all facilities and help for smooth progress of the project. 
We are thankful and fortunate enough to get constant encouragement, support and guidance from all teaching staffs of Department of Computer Engineering which helped us in successfully starting our project. At last we would like to thank all the unseen authors of various articles on the internet, helping us become aware of the research currently ongoing in this field and all our colleagues for providing help and support in our work.

\section{REFERENCES}

[1] Rani, L. Paul Jasmine, et al. "Dynamic traffic management system using infrared (IR) and Internet of Things (IoT)." 2017 Third International Conference on Science Technology Engineering \& Management (ICONSTEM). IEEE, 2017.

[2] Al-Khateeb, Khalid, and Jaiz AY Johari. 'Intelligent dynamic traffic light sequence using RFID." 2008 International Conference on Computer and Communication Engineering. IEEE, 2008.

[3] JagannadhaRao, P., et al. "Thermal Based Intelligent Traffic Light Control System." International Journal of Engineering Science and Emerging Technologies Aug-2012 3.1 (2012).

[4] Riyazhussain, Sk, et al. "Raspberry pi controlled traffic density monitoring system." 2016 International Conference on Wireless Communications, Signal Processing and Networking (WiSPNET). IEEE, 2016.

[5] Younes, Maram Bani, and Azzedine Boukerche. "An intelligent traffic light scheduling algorithm through VANETs." 39th Annual IEEE Conference on Local Computer Networks Workshops. IEEE, 2014.

[6] Li, Jinyang, Yuanrui Zhang, and Yixiang Chen. "A self-adaptive traffic light control system based on speed of vehicles." 2016 IEEE International Conference on Software Quality, Reliability and Security Companion (QRS-C). IEEE, 2016.

[7] El-Tantawy, Samah, and Baher Abdulhai. "An agent-based learning towards decentralized and coordinated traffic signal control." 13th International IEEE Conference on Intelligent Transportation Systems. IEEE, 2010.

[8] Wei, Hua, et al. "Intellilight: A reinforcement learning approach for intelligent traffic light control." Proceedings of the 24th ACM SIGKDD International Conference on Knowledge Discovery Data Mining. 2018.

[9] Smith, Stephen F., et al. "Smart urban signal networks: Initial application of the surtrac adaptive traffic signal control system." Twenty-third international conference on automated planning and scheduling. 2013. 